\title{
AVALIAÇÃO ESPACIAL DA QUALIDADE DA ÁGUA DO RIO CATOLÉ GRANDE
}

\author{
Camila Andrade Coqueiro Moraes ${ }^{1}$, José Luis Rodrigues Lima ${ }^{2}$, Carlos Leôncio \\ Souza Costa ${ }^{2}$, Romário Oliveira de Santana ${ }^{2}$, Flávia Mariani Barros ${ }^{3}$ \\ 1 Graduanda em Engenharia Ambiental pela Universidade Estadual do Sudoeste da \\ Bahia (milacoqueiro@hotmail.com), Itapetinga-Brasil \\ 2 Graduando em Engenharia Ambiental pela Universidade Estadual do Sudoeste da \\ Bahia \\ 3 Professora Doutora do Departamento de Ciências Exatas e Naturais da \\ Universidade Estadual do Sudoeste da Bahia
}

\section{Recebido em: 08/09/2015 - Aprovado em: 14/11/2015 - Publicado em: 01/12/2015 DOI: http://dx.doi.org/10.18677/Enciclopedia Biosfera 2015100}

\section{RESUMO}

A disponibilidade da água é relacionada com o clima e as características físicas e biológicas da bacia hidrográfica, sendo que as atividades industriais, agroindustriais e urbanas contribuem fortemente para sua degradação. Em Itapetinga-BA, o processo de industrialização contribui para a deterioração da qualidade das águas do Rio Catolé Grande. Desta forma, o objetivo deste trabalho foi caracterizar um trecho deste rio, observando os efeitos do lançamento de efluentes ou outros resíduos por meio dos valores de: $\mathrm{pH}$; temperatura; oxigênio dissolvido; condutividade elétrica; turbidez; sólidos totais, fixos e voláteis. Para tanto foram coletadas amostras na calha central do rio em três diferentes pontos: antes, durante e após o perímetro urbano. As amostras foram levadas em seguida para o laboratório, onde foram medidas as variáveis. Os valores de $\mathrm{pH}$, oxigênio dissolvido e porcentagem de saturação de oxigênio (PSO) foram diminuindo de um ponto para o outro, o contrário ocorreu para a temperatura. As demais variáveis analisadas (condutividade elétrica, turbidez, sólidos totais, fixos e voláteis) não apresentaram diferenças significativas entre os pontos a $5 \%$ de probabilidade pelo teste de Tukey.

PALAVRAS-CHAVE: Monitoramento ambiental, poluição, recursos hídricos.

\section{SPACE AVALIATION OF WATER QUALITY OF THE CATOLÉ GRANDE RIVER}

\begin{abstract}
Water availability is linked to the climate, physical and biological characteristics of the river basin, and the industrial, agro-industrial and urban activities contribute to its degradation. The industrialization process contributes to the deterioration of water quality of the Catolé Grande river located in Itapetinga-BA. Thus, the aim of this study was to characterize a river stretch, observing the effects of the discharge of effluents and other wastes through: $\mathrm{pH}$; temperature; dissolved oxygen; conductivity; turbidity; total, fixed and volatile solids. Samples were collected in the central channel of the river at three different points: before, during and after the urban perimeter. The samples were sent to laboratory for analyses. The $\mathrm{pH}$, dissolved oxygen and oxygen percent saturation (PSO) were decreasing from one point to another, while the opposite was observed to temperature. The other analyzed variables (conductivity,


turbidity, total, fixed and volatile solids) showed no significant differences between points at $5 \%$ probability by Tukey test.

KEYWORDS: Environmental monitoring, pollution, water resources.

\section{INTRODUÇÃO}

A qualidade dos recursos naturais, como a água, tem relação com a saúde humana e, por isso, a sociedade tem direcionado a atenção para as suas atividades, produtos ou serviços e os impactos ambientais negativos gerados por eles (MOURA et al., 2013).

A qualidade da água é resultante da relação entre os fenômenos naturais e os antrópicos e sua disponibilidade gira em torno do clima, das características físicas e biológicas da bacia hidrográfica. Como as atividades industriais, agroindustriais e urbanas alteram as características da bacia, (MORUZZI et al., 2012), salienta-se a importância de monitorar a qualidade da água com o objetivo de promover a sustentabilidade dos recursos e o desenvolvimento econômico (MARCONATI SANTI et al., 2012).

Tendo como referência o município de Itapetinga na Bahia, nota-se que o processo de industrialização promoveu o desenvolvimento econômico da cidade e seu crescimento populacional, entretanto, contribuiu para a deterioração da qualidade das águas do rio que corta o município através dos despejos de efluentes domésticos e industriais.Desta forma, este trabalho tem como objetivo caracterizar um trecho do Rio Catolé Grande antes, durante e após o perímetro urbano.

\section{MATERIAL E MÉTODOS}

As amostras de água foram coletadas em três pontos distintos do rio Catolé Grande no município de Itapetinga, Bahia (Figura 1). O Ponto 1 (P1) está situado à montante da cidade, acima do local de captação de água do Serviço Autônomo de Água e Esgoto (SAAE), suas coordenadas geográficas são: Latitude: 15014'35", Longitude 40 $10^{\circ} 49^{\prime \prime}$ e Altitude: $250 \mathrm{~m}$. O Ponto 2 (P2) se encontra na Avenida das Indústrias no Bairro da Nova Itapetinga, com coordenadas geográficas: Latitude: 15 $15^{\prime} 04^{\prime \prime}$, Longitude 4014'22" e Altitude: 248 m. O Ponto 3 (P3) está localizado em uma propriedade na zona rural do município, e suas coordenadas geográficas são: Latitude: $15^{0} 16^{\prime} 09^{\prime \prime}$, Longitude 4013'04" e Altitude: 243 m.

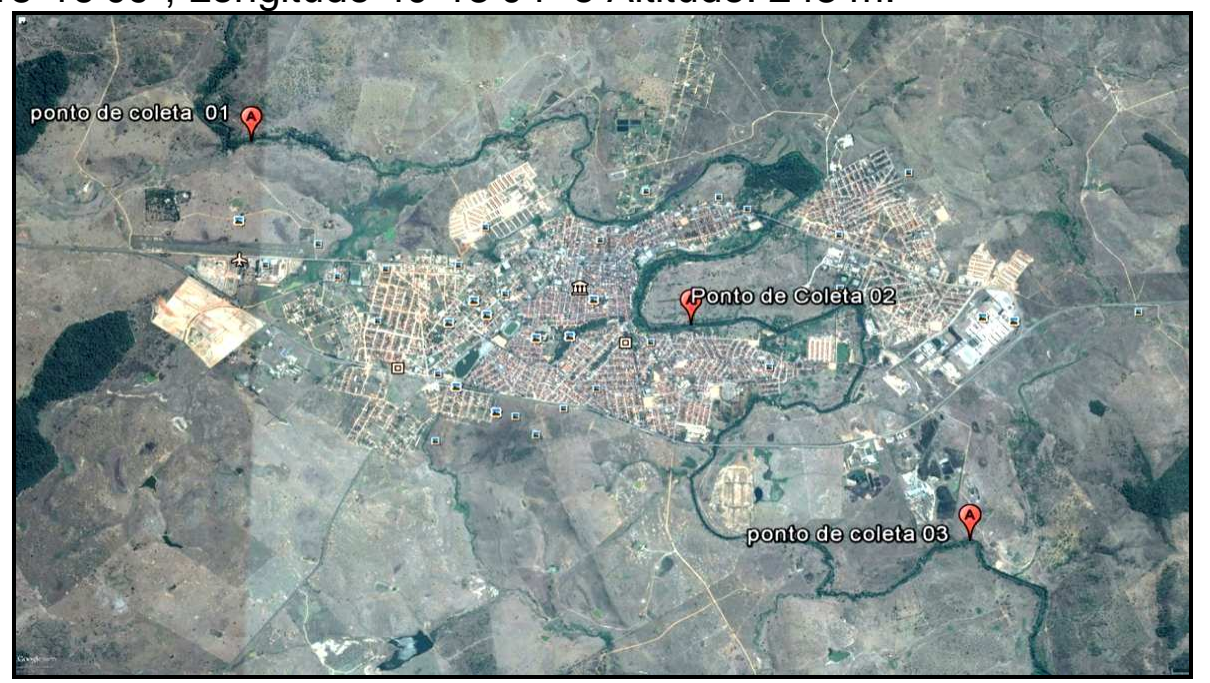

FIGURA 1 - Foto da cidade de Itapetinga-BA com a indicação dos pontos de coleta no Rio Catolé Grande (Fonte: Google). 
As amostras foram coletadas em cada um dos três pontos, na calha central do rio e profundidade de 15 a $30 \mathrm{~cm}$ em três repetições, segundo metodologia descrita em CETESB (1988). Em seguida foram encaminhadas para o Laboratório de Dispersão de Poluentes da Universidade Estadual do Sudoeste da Bahia (UESB) em Itapetinga-BA para a realização das análises. Em cada amostra de água foram realizadas as análises de: oxigênio dissolvido; condutividade elétrica; turbidez; pH; sólidos totais, fixos e voláteis.

$O$ oxigênio dissolvido (OD) foi quantificado por meio do método da modificação de Winkler modificado pela azida sódica. A condutividade elétrica (CE) foi mensurada por meio do condutivímetro da marca Digimed, modelo DM-32. A determinação da turbidez foi realizada por meio de turbidímetro da marca Digimed, modelo DM-TU. O pH foi obtido utilizando-se pHmetro da marca Digimed, modelo DM-22. Os sólidos totais, fixos e voláteis foram determinados pelo método gravimétrico. Todas as análises realizadas se basearam nas metodologias descritas em MATOS (2004).

\section{RESULTADOS E DISCUSSÃO}

Na Figura 2 estão apresentados os resultados das variáveis $\mathrm{pH}$, temperatura, OD e PSO dos pontos P1, P2 e P3.
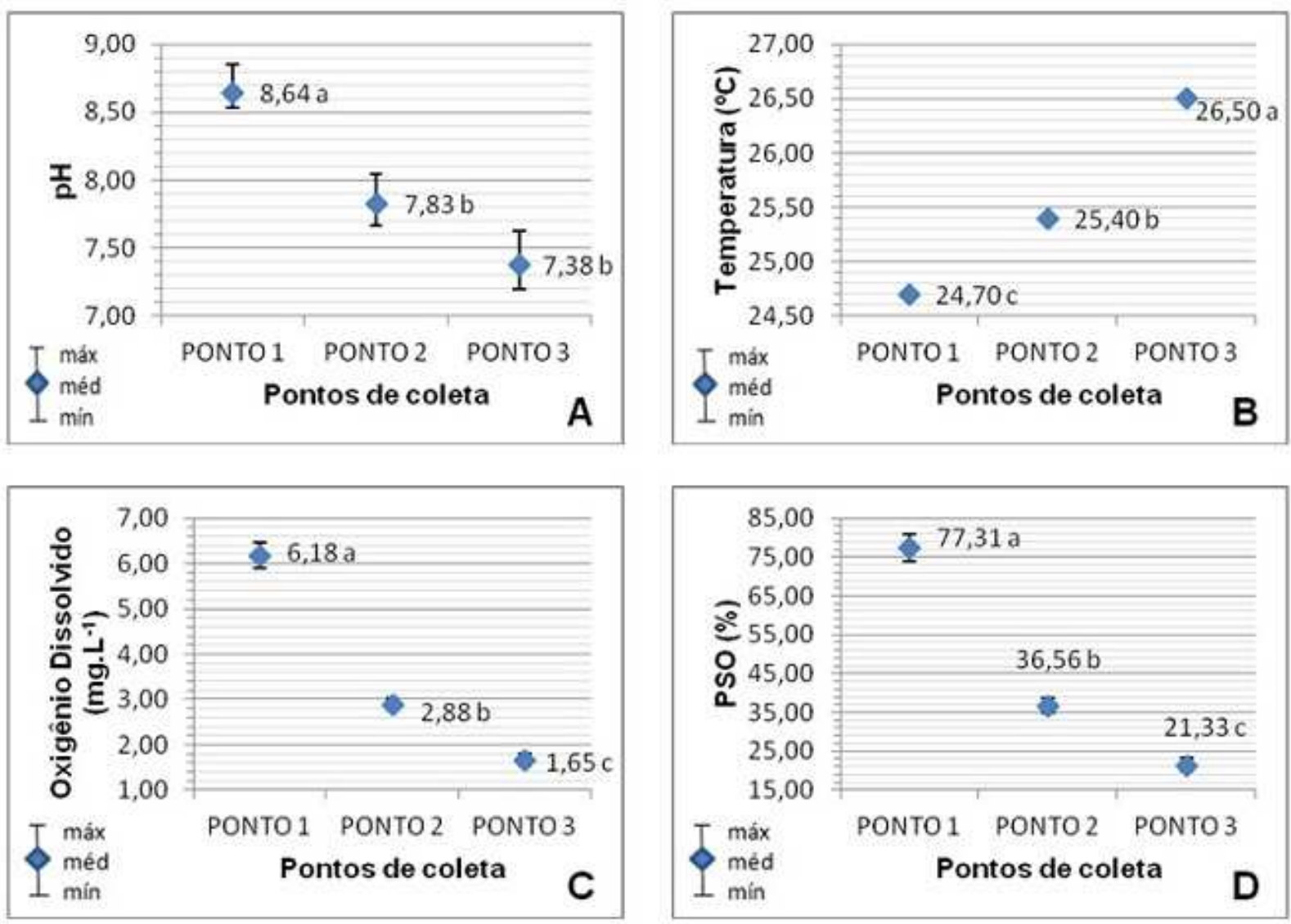

FIGURA 2 - Valores das variáveis de qualidade da água: (A) pH; (B) temperatura; (C) oxigênio dissolvido; (D) PSO; em função dos pontos de coleta, no rio Catolé Grande, Bahia (Fonte: Elaborada pelos autores). ${ }^{(1)}$ 
$\mathrm{O} \mathrm{pH}$ variou de 7,38 a 8,64 entre os 3 pontos de coleta, porém comparando os valores médios obtidos para cada ponto (Figura $2 \mathrm{~A}$ ) observou-se que a $5 \%$ de probabilidade pelo teste de Tukey o $\mathrm{P} 1$ foi o único que diferiu significativamente dos outros, apresentando o valor maior.

Os valores de $\mathrm{pH}$ foram diminuindo conforme os pontos de coleta ficavam mais próximos à cidade e suas indústrias (Figura 2A), apontando para uma elevação da carga orgânica oriunda do lançamentos de efluentes. Segundo NOZAKI et al. (2014), o pH é influenciado pela quantidade de matéria orgânica a ser decomposta, sendo inversamente proporcional a este fator, ou seja, quanto mais material a ser decomposto, mais dióxido de carbono e ácidos fracos são gerados para auxiliar a sua decomposição (fermentação) e respiração dos organismos, tornando consequentemente, menor o $\mathrm{pH}$ do meio, corroborando com os resultados obtidos neste trabalho.

Os valores de $\mathrm{pH}$ dos três pontos estudados estão dentro do limite proposto pela Resolução CONAMA 357 (BRASIL, 2005), onde os valores devem estar situados entre 6,0 e 9,0 para qualquer condição de qualidade de água doce.

A temperatura média da água diferiu significativamente pelo teste de Tukey a $5 \%$ de probabilidade (Figura $2 \mathrm{~B}$ ) nos três pontos avaliados, sendo o menor valor encontrado no $\mathrm{P} 1\left(24,7^{\circ} \mathrm{C}\right)$, seguido do $\mathrm{P} 2\left(25,4^{\circ} \mathrm{C}\right)$ e P3 $\left(26,5^{\circ} \mathrm{C}\right)$, respectivamente. Possivelmente esta variação está relacionada com o nível de cobertura vegetal de cada lugar alternando a exposição aos raios solares e a contribuição de efluentes domésticos e industriais.

Os valores de oxigênio dissolvido diferiram significativamente entre si pelo teste de Tukey a $5 \%$ de probabilidade (Figura $2 \mathrm{C}$ ) nos três pontos avaliados, sendo de $6,18 \mathrm{mg} \cdot \mathrm{L}^{-1}, 2,88 \mathrm{mg} \cdot \mathrm{L}^{-1}$ e $1,65 \mathrm{mg} \cdot \mathrm{L}^{-1}$, respectivamente para $\mathrm{P} 1, \mathrm{P} 2$ e $\mathrm{P} 3$. O oxigênio dissolvido é indispensável para os organismos aeróbios aquáticos e, por isso, é considerado como um dos principais parâmetros indicadores da poluição das águas, sendo que seus baixos níveis indicam a presença de matéria orgânica, geralmente proveniente de efluentes (OLIVEIRA \& SILVA, 2014), como nos casos do P2 e P3 do presente trabalho, onde os valores de OD foram baixos devido à contribuição de efluentes ricos em material orgânico.

Apenas o valor de OD do P1 se encontra dentro do limite estabelecido pela Resolução CONAMA 357 (BRASIL, 2005), a qual determina que as amostras não devem possuir concentrações de OD inferiores a $6 \mathrm{mg} \cdot \mathrm{L}^{-1}, 5 \mathrm{mg} \cdot \mathrm{L}^{-1}$ e $4 \mathrm{mg} \cdot \mathrm{L}^{-1}$, referentes às condições das classes 1 a 3 para águas doces, respectivamente.

Segundo VON SPERLING (1996) o aumento da temperatura diminui a solubilidade de gases. Neste caso, ao comparar os valores de temperatura e OD (Figuras 2B e 2C), isto se confirma, onde o ponto com a temperatura maior possui o menor valor de OD. A água quando livre da poluição orgânica possui um limite da concentração de oxigênio dissolvido relacionado a sua temperatura e pressão atmosférica local denominada concentração de saturação de oxigênio dissolvido (PORTO et al., 1991).

A partir desta concentração é possível calcular a porcentagem de saturação do oxigênio para observar a capacidade de autodepuração de um corpo d'água. Neste caso, os valores médios foram $77,31 \%, 36,56 \%$ e $21,33 \%$ em P1, P2 e P3, respectivamente, como pode ser observado na Figura 2D, sendo que todos diferiram a $5 \%$ de probabilidade pelo teste de Tukey. 
Na Figura 3 estão apresentados os resultados das variáveis CE e turbidez dos pontos P1, P2 e P3.
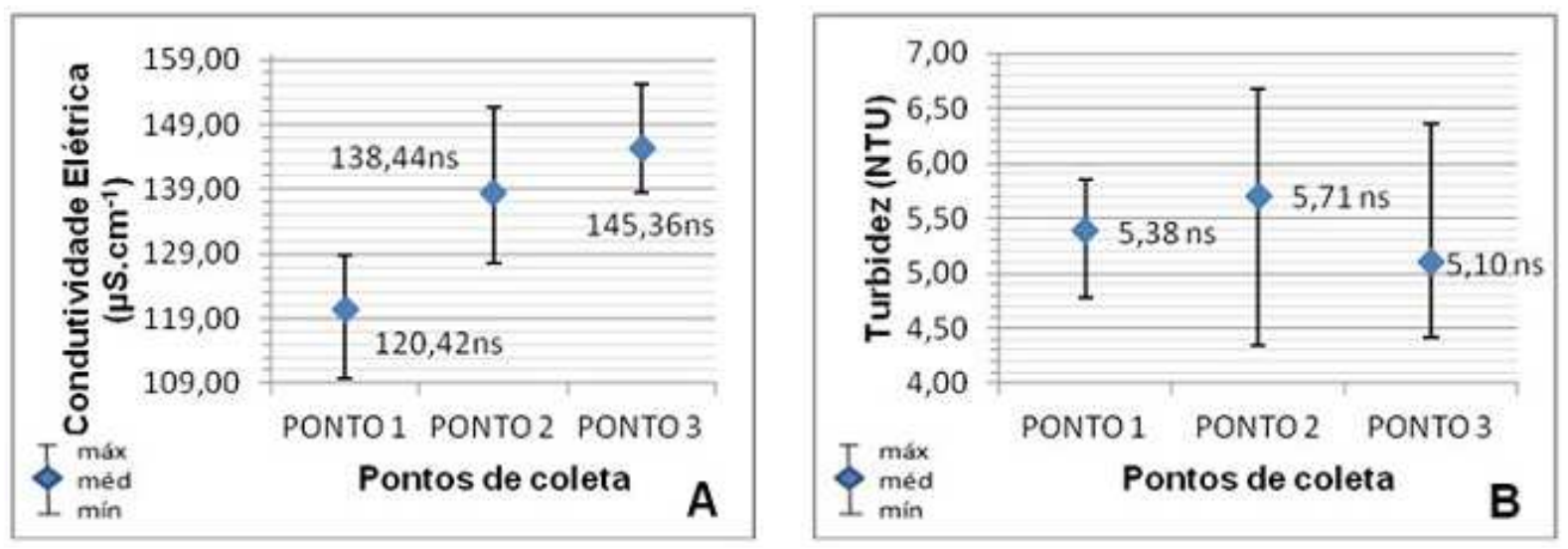

FIGURA 3 - Valores das variáveis de qualidade da água: (A) condutividade elétrica; e (B) turbidez; em função dos pontos de coleta, no rio Catolé Grande, Bahia (Fonte: Elaborada pelos autores). ${ }^{(2)}$

Os valores médios de condutividade elétrica encontrados foram: 120,42 $\mu S . \mathrm{cm}^{-1}, 138,44 \mu \mathrm{S} . \mathrm{cm}^{-1}$ e $145,36 \mu S . \mathrm{cm}^{-1}$ para os pontos P1, P2 e P3, respectivamente (Figura 3A). Segundo IZARIAS et al. (2014), a concentração de substâncias ionizadas dissolvidas é responsável pelo valor da condutividade elétrica e esta é resultante do lançamento de resíduos industriais ou domésticos, fato este que corrobora com os resultados obtidos no presente trabalho, com aumento da CE nos locais mais impactados por efluentes.

O valor médio encontrado para turbidez em todos os pontos foi próximo a 5 NTU (Figura 3B), especificamente 5,38 NTU, 5,71 NTU e 5,10 NTU nos pontos P1, P2 e P3, respectivamente. Este parâmetro representa a alteração que a luz sofre ao atravessar a água, provocada por partículas suspensas no meio, compostas por material coloidal e organismos microscópicos, como o plâncton (KLUSKA et al., 2014). Em nenhum ponto avaliado foi ultrapassado o valor limite permitido pela Resolução CONAMA 357 (BRASIL, 2005) para turbidez, que é de 40 UNT para a condição de qualidade de águas doces da classe 1.

$\mathrm{Na}$ Figura 4 estão apresentados os resultados de sólidos totais, fixos e voláteis encontrados nos pontos P1, P2 e P3. 

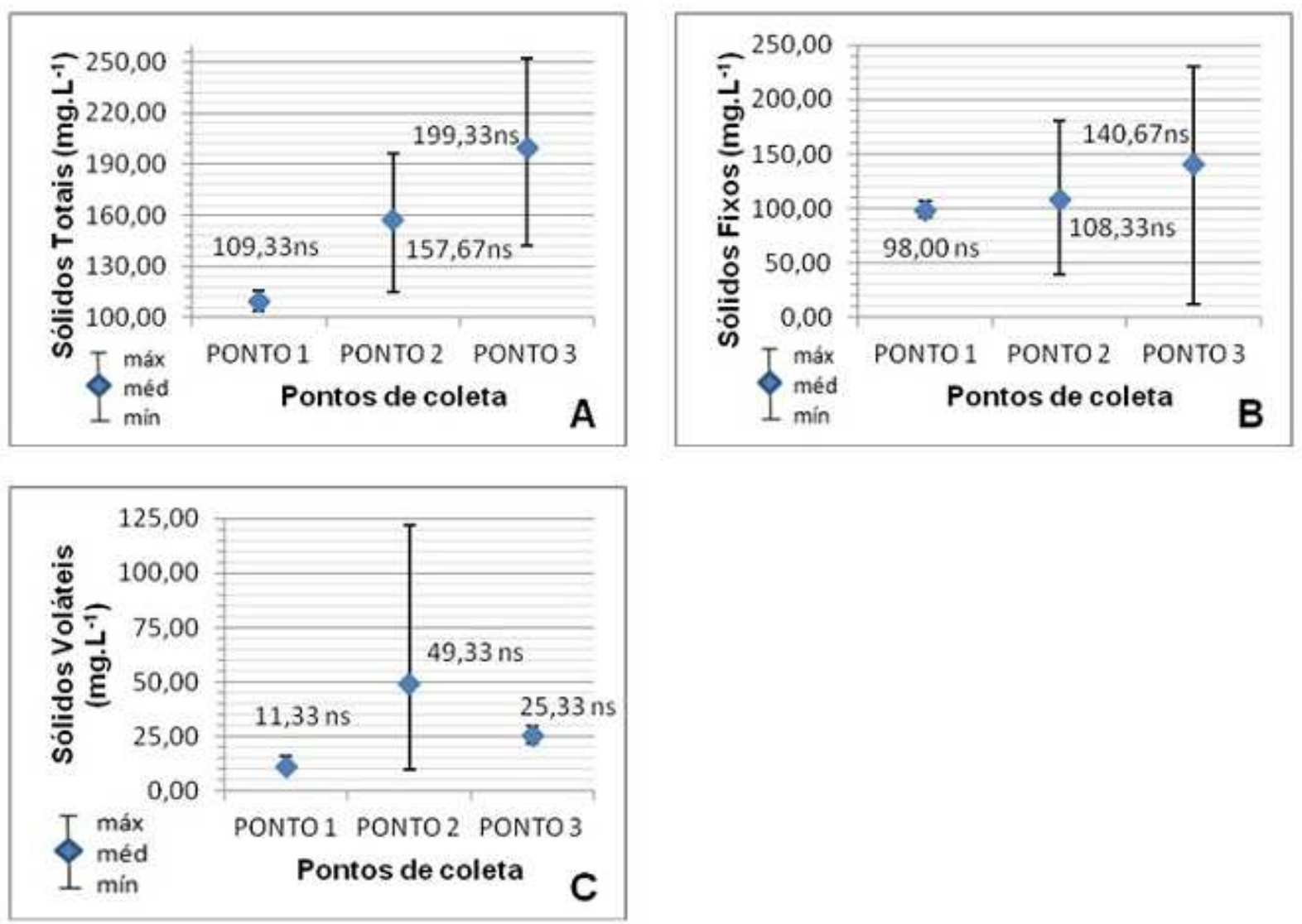

FIGURA 4 - Valores das variáveis de qualidade da água: (A) sólidos totais; (B) sólidos fixos; e (C) sólidos voláteis; em função dos pontos de coleta, no rio Catolé Grande, Bahia (Fonte: Elaborada pelos autores). ${ }^{(3)}$

Os sólidos são constituídos por todas as impurezas encontradas na água, com exceção dos gases dissolvidos, interferindo nas características do meio, conforme destacado por MACÉDO (2001).

Neste estudo foram encontrados valores de sólidos totais, fixos e voláteis, respectivamente, de $109,33 \mathrm{mg} \cdot \mathrm{L}^{-1}, 98,00 \mathrm{mg} \cdot \mathrm{L}^{-1}$ e $11,33 \mathrm{mg} \cdot \mathrm{L}^{-1}$ para o $\mathrm{P} 1,157,67$ $\mathrm{mg} \cdot \mathrm{L}^{-1}, 108,33 \mathrm{mg} \cdot \mathrm{L}^{-1}$ e $49,33 \mathrm{mg} \cdot \mathrm{L}^{-1}$ para o P2, e de 199,33 mg. $\mathrm{L}^{-1}, 140,67 \mathrm{mg} \cdot \mathrm{L}^{-1} \mathrm{e}$ 25,33 mg.L-1 para o P3 (Figuras 4A, 4B e 4C). Nenhum dos valores de sólidos observados apresentou diferença entre os pontos avaliados pelo teste de Tukey a $5 \%$ de probabilidade de erro.

BUZELLI \& CUNHA-SANTINO (2013) encontraram valores semelhantes de sólidos totais para o reservatório de Barra Bonita-SP, sendo de 100,00 $\pm 6,00 \mathrm{mg}^{-\mathrm{L}^{-1}}$ para o período de estiagem e $156,00 \pm 31,00 \mathrm{mg}^{-\mathrm{L}^{-1}}$ para o período de chuvas, reforçando os resultados encontrados nesse trabalho para ambientes aquáticos expostos a atividades antrópicas.

Como explicado por COELHO (2013), é esperado que as frações totais de variáveis de qualidade sejam mais estáveis, pois, por se tratarem de valores globais, dependem de menos fatores que suas frações, variando em função da contribuição de cargas, como visto neste estudo. 


\section{CONCLUSÃO}

Tendo como base os resultados obtidos e considerando-se as condições em que o estudo foi realizado, conclui-se que: o pH variou entre 7,38 e 8,64 e o oxigênio dissolvido entre $1,65 \mathrm{mg} \cdot \mathrm{L}^{-1}$ e $6,18 \mathrm{mg} \cdot \mathrm{L}^{-1}$, apresentando diminuição dos valores a medida que o rio fluía a jusante do ponto 1 . A temperatura variou entre $24,7^{\circ} \mathrm{C}$ e $26,5^{\circ} \mathrm{C}$; a condutividade elétrica variou entre $120,42 \mu \mathrm{S} . \mathrm{cm}^{-1}$ e $145,36 \mu \mathrm{S} . \mathrm{cm}^{-1}$; os sólidos totais variaram de $109,33 \mathrm{mg} \cdot \mathrm{L}^{-1}$ a $199,33 \mathrm{mg} \cdot \mathrm{L}^{-1}$; os sólidos fixos variaram de $98,00 \mathrm{mg} \cdot \mathrm{L}^{-1}$ a $140,67 \mathrm{mg} \cdot \mathrm{L}^{-1}$, aumentando do ponto menos poluído (P1) para os mais poluídos (P2 e P3). Enquanto a turbidez variou de 5,10 NTU a 5,71 NTU; e os sólidos voláteis variaram de $11,33 \mathrm{mg} \cdot \mathrm{L}^{-1}$ a $49,33 \mathrm{mg} \cdot \mathrm{L}^{-1}$.

Através dos valores dos parâmetros tidos como significativos pela análise estatística, foi possível observar a variação espacial da qualidade da água do rio Catolé Grande, mostrando uma deterioração causada por possíveis contribuições ao longo do perímetro urbano.

\section{REFERÊNCIAS}

BRASIL. CONSELHO NACIONAL DO MEIO AMBIENTE-CONAMA. Resolução CONAMA (Conselho Nacional de Meio Ambiente). Resolução n.․ 357, de 17 de Março de 2005. Dispõe sobre a classificação dos corpos de água e diretrizes ambientais para o seu enquadramento, bem como estabelece as condições e padrões de lançamento de efluentes, e dá outras providências. Diário Oficial da República Federativa do Brasil, Brasília, 2005.

BUZELLI, G.M.; CUNHA-SANTINO, M.B. Análise e diagnóstico da qualidade da água e estado trófico do reservatório de Barra Bonita, SP. Revista Ambiente \& Água, Taubaté, v.8, n.1, 2013.

CETESB (Companhia de Tecnologia de Saneamento Ambiental do estado de São Paulo). Guia de coleta e preservação de amostras de água. São Paulo, CETESB, 1988.

COELHO, M. Estratégia de monitoramento da qualidade da água para a gestão de recursos hídricos em bacias urbanas (Dissertação de mestrado). Curitiba: Universidade Federal do Paraná, 2013. 161 p.

IZARIAS, N. S.; PERANZONI, A. C. M.; KAFER, G. A.; OLIVEIRA, A. M.; SOUZA, A. S.; OLIVEIRA, E. N.; SANTANA, E. R. Qualidade das águas em áreas urbanas do Rio Taquari nos municípios de Estrela e Lajeado - RS. Ciência e Natura , v. 36, p. 789-797, 2014.

KLUSKA, M.; ALMEIDA, S. M. Z.; ALMEIDA, L. P. Caracterização ambiental e análise da água superficial do percurso urbano do Rio Xanxerê. Unoesc \& Ciência ACET, Joaçaba, v. 5, n. 1, p. 31-38, 2014.

MACÊDO, J. A. B. Águas \& águas. São Paulo: Varela, 2001, 505p. Minerais do Paraná SA. (MINEROPAR). 
MARCONATI SANTI, G.; FURTADO, C. M.; MENEZES, R. S.; KEPELLER, E. C. Variabilidade espacial de parâmetros e indicadores de qualidade da água na subbacia hidrográfica do Igarapé São Francisco, Rio Branco, Acre, Brasil. Ecol. apl., Lima, v. 11, n. 1, 2012.

MATOS, A. T. de. Práticas de qualidade do meio físico e ambiental. Viçosa: AEAGRI. (Série Caderno Didático 34). 64p. 2004.

MORUZZI, R. B.; CONCEIÇÃO, F. T.; SARDINHA, D. S.; HONDA, F. P.; NAVARRO, G. R. B. Avaliação de cargas difusas e simulação de autodepuração no Córrego da Água Branca, Itirapina (SP). Geociênc. (São Paulo), São Paulo, v. 31, n. 3, 2012.

MOURA, V. M.; BRITO, S. M. O.; SILVA, A. B. Avaliação dos Parâmetros Indicadores da Qualidade da Água para Verificar o Estado de Conservação das Represas do Rio Ipitanga, Salvador, BA, Brasil. Revista Virtual de Química , v. 5, p. 870-886, 2013.

NOZAKI, C. T.; MARCONDES, M. A.; LOPES, F. A.; SANTOS, K. F.; LARIZZATTI, P. S. C. Comportamento temporal de oxigênio dissolvido e ph nos rios e córregos urbanos. ATAS DE SAÚDE AMBIENTAL - ASA , v. 2, n. 1, p. 29, 2014.

OLIVEIRA, L. N.; SILVA, C. E. Qualidade da água do Rio Poti e suas implicações para atividade de lazer em Teresina-PI. Revista Equador, v. 3, n. 1, p. 128-128, 2014.

PORTO, F.A.; BRANCO, S.M.; LUCA, S.J. Caracterização da qualidade da água. In: PORTO, R.L.(Org.). Hidrologia ambiental, São Paulo: EDUSP, p.375-390, 1991.

VON SPERLING, M. V. Introdução a qualidade das águas e ao tratamento de esgotos. Belo Horizonte - MG. p. 243,1996. 